\title{
Rasmussen's Aneurysm: A Case Report
}

\section{Karhate $\mathbf{M}^{*}$, Senhaji L, Amara B and Serraj M}

\section{CHU Hassan II Fès, Morocco}

*Corresponding author: Karhate M, CHU Hassan II Fès, Morocco, Tel: 212662765080, E-mail: meryem.karhate@ gmail.com; meryem.andaloussi01@gmail.com

\section{Citation: Karhate M, Senhaji L, Amara B, Serraj M (2019) Rasmussen's Aneurysm: A Case Report. J Case Rep} Stud 7(4): 405. doi: 10.15744/2348-9820.7.405

Received Date: November 23, 2018 Accepted Date: August 27, 2019 Published Date: August 29, 2019

\begin{abstract}
Background: Aneurysm is due to erosion of the pulmonary artery wall by the inflammatory granuloma. Originally described since the 18 th century, this aneurysm has become rare with the advent of anti-bacillary drugs.

Case presentation: A 46-year-old patient with no significant pathological history, suffering from hemoptysis of great abundance for the past 4 years, evolving in a context of general state preservation. A clinical examination is without particularity. A remote angio-CT of the bleeding episode revealed lower lobar branch aneurysm of the left pulmonary artery that is partially thrombosed. A check-up was requested for a bronchial endoscopy with a search for tubercle bacilli in the fibro-aspiration fluid, which returned negative. A diagnostic report seeking an etiological diagnosis of vasculitis is negative. The patient has benefited from an arterial embolization that is complicated after 1 month by hemoptysis of average abundance hence the indication for surgical exploration.

Conclusion: During preoperative exploration, the opening of the fissure resulted in the opening of an aneurysm. A pneumonectomy was performed. Pathological examination has returned to favor a mycotic tubercular aneurysm of the pulmonary artery associated with cause follicular pulmonary tuberculosis. The patient is put on anti-bacillary treatment with good evolution.
\end{abstract}

Keywords: Hemoptysis; Rasmussen's Aneurysm; Tuberculosis

\section{Case presentation}

This is a 46-year-old male patient, never treated for tuberculosis, no recent contact with tuberculosis, no history of smoking or specific exposure to the toxic product, no notion of long-term drug use, no notion of heart disease or other respiratory or extralung pathology. Who for 4 years has had recurrent episodes of hemoptysis of great abundance evolving in a context of preservation of the general state without other associated respiratory or extra respiratory signs.

The clinical examination finds a patient in good general condition, slightly discolored conjunctiva, eupneic, with a respiratory rate of 18 cycles per minute, oxygen saturation in the open air at 97\%, bilateral fine crepitation at the two pulmonary bases, the cardiovascular examination as well as the rest of the clinical examination is without particularity

A frontal chest X-ray was performed (Figure 1), supplemented by a thoracic angio CT (Figure 2). The following diagnoses were mentioned:

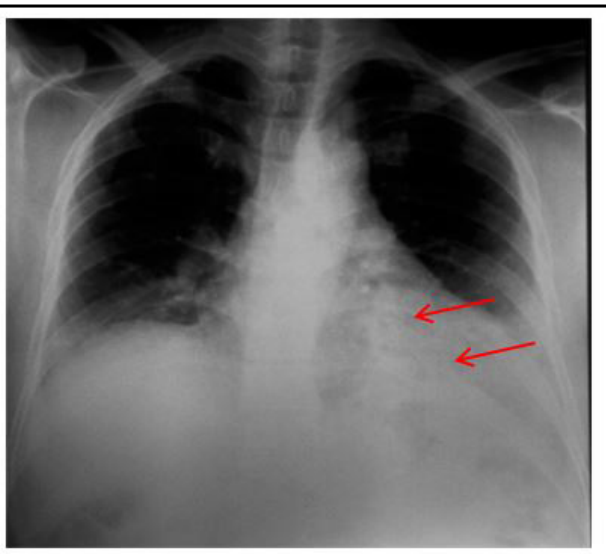

Figure 1: Front Chest X-Ray Shows a Foyer at the Lower Half of the Left Thoracic Hemi-Field,

Which Erases the Left Edge of the Heart, Associated With a Left Retrocardiac Alveolar Opacity 


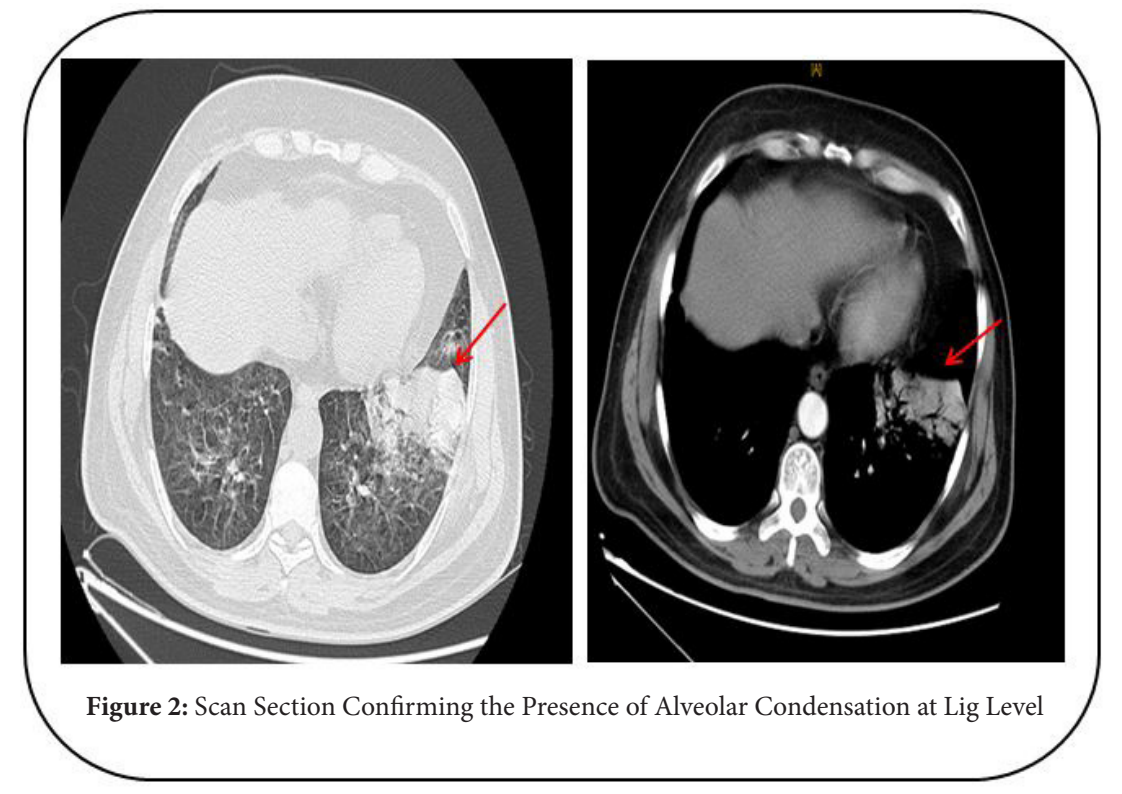

- Lung sequestration

- Chronic pneumonia

- Pneumonic tuberculosis

Bronchial fibroscopy performed the day after his hospitalization showed a deformation of the bronchial tree with thickened spurs to the right. On the left is an edema of the mucous membranes, bleeding from the basal pyramid whose spur is thickened and whose orifice is unheterisable.

Bronchial biopsies have returned in favour of non-specific inflammatory remodeling.

The search for tubercle bacilli in the sputum and in the fibro-aspiration fluid came back negative.

A second thoracic CT angiogram was performed 3 days after hemoptysis control (Figure 3).

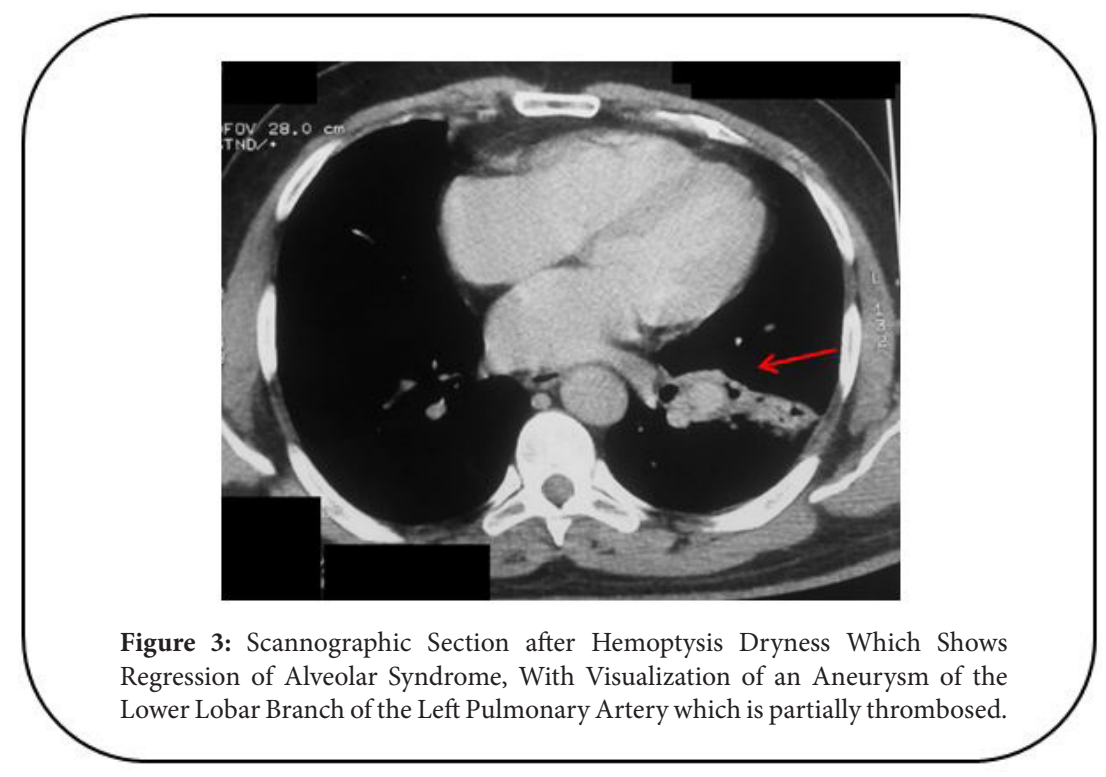

In view of the radiological aspect, the following diagnoses were mentioned:

Vasculitis in the context of tuberculosis or Behçet's disease, or syphilis.

A vasculitis examination was requested: repeated questions with the patient showed an absence of oral or genital aphthosis, or a previous thromboembolic event or other extra respiratory signs. Syphilitic serologies were performed with negative results, as well as cytoplasmic anti-neutrophil antibodies (ANCA).

CT pulmonary angiogram (CTPA) performed 10 days after the last hemorrhagic episode showed a regression of alveolar syndrome, with visualization of an aneurysm of the lower lobar branch of the left pulmonary artery that is partially thrombosed. 
The patient received arterial embolization following the angiography result, which was complicated after 1 month by hemoptysis of repetitive mean abundance, not controlled by medical treatment, hence the indication for surgical exploration.

The patient was readmitted one month after discharge and referred for thoracic surgery. Intraoperative exploration showed a bluish appearance of the lower lobe, the opening of the fissure was impossible due to the presence of aneurysmal dilation of the pulmonary artery at arterial level X after the origin of the Nelsonian artery. After placing the pulmonary artery in the hilum on the lake and ligating the Nelsonian artery, the opening of the rest of the fissure leads to the opening of an aneurysm where the embolization material is found. There was no possibility of partial resection, extrapericardial pneumonectomy was performed.

The pathological anatomy of pneumectomy has returned in favour of a mycotic tubercular aneurysm of the pulmonary artery associated with caseofollicular pulmonary tuberculosis.

\section{Diagnosis}

Rasmussen's aneurysm

\section{Comments}

The patient was treated with anti-bacillary treatment with a good evolution, he no longer had post-operative hemoptysis, and the search for tubercle bacilli in the sputum was negative. Patient has been declared cured.

\section{Discussion}

Tuberculosis remains common in Western countries and the pulmonary form of the disease is a common cause of hemoptysis [1]. Hemoptysis in cases of tubercular sequelae is most often of bronchial arterial origin. In the acute phase, pulmonary arterial origin is observed in cases of false Rasmussen aneurysms [1].

First described by Rasmussen in 1868, the false aneurysm of the pulmonary artery complicating tuberculosis has become increasingly rare with the widespread use of antibiotics [2].

The term Rasmussen's pseudo aneurysm refers specifically to tuberculous etiology. It results from a pulmonary tuberculosis cavitary lesion that erodes the adjacent structures in the lung, when a branch of the pulmonary artery come adjacent to or within the cavity, its wall progressively weaken as granulation tissue replaces both the adventitia and the media layer. Thereafter, the artery wall expands until it bursts resulting in pseudo aneurysm formation. These pseudo aneurysms can potentially rupture producing massive hemoptysis [3].

In about $80 \%$ of cases, a bronchial or other systemic artery is the cause of the bleeding, but in the remaining $20 \%$, a pulmonary arterial origin is found [4].

Rasmussen aneurysm rupture has a reported incidence of $84 \%$ and a mortality rate greater than $80 \%[3,5]$.

Auerbach et al., published a series of autopsies of 1114 pulmonary tuberculosis patients with cavitary lesions in 1939 and found 45 cases of pulmonary arterial aneurysm (4\%), 38 of which were responsible for fatal hemoptysis [3].

Similarly, Plessinger et al., published a series of 56 cases of Rasmussen's aneurysm in 1956, 47 (87\%) of which were caused by massive pulmonary arterial bleeding [5].

In 1984, out of a prospective series of 189 patients treated for massive hemoptysis, Remy et al. found only 5 cases related to pulmonary artery rupture, of which only 2 were related to cavitary tuberculosis [6].

In 2005, Sbano F et al., reported 8 cases of pulmonary artery rupture in 76 patients examined for massive hemoptysis, including 5 cases of chronic tuberculosis [7].

Although this pathology has become rare, it remains responsible for significant lethality due to the severity of associated hemoptysis and requires special management [1].

In countries with a high prevalence of tuberculosis despite therapeutic success, it seems justified to look for a tubercular or systemic origin in the presence of massive hemoptysis depending on the clinical, radiological or bacteriological orientation; however, in the absence of any sign of orientation, diagnosis remains difficult.

The injected multicut scanner is the reference examination for the etiological and pathophysiological diagnosis of hemoptysis and guides the clinician on the type of angiography to be performed: either bronchial arteriography or selective pulmonary angiography. This key examination must be performed strictly with reconstructions of indigenous axial views by the radiologist performing it. In 2008, Khalil et al., revealed the value of performing a multi-cut pulmonary CT scan injected before any endovascular treatment to avoid ignoring a pulmonary arterial origin [8].

Aneurysms should be particularly sought on beaches of parenchymal necrosis. 
In 2018 Vatturi Venkata Satya Prabhakar Rao report multiple giant bilateral pseudo aneurysms of pulmonary artery, also known as Rasmussen's aneurysms, which remained silent and unrevealed despite the large size and multiplicity unearthed by fluorine-18 fluorodeoxyglucose positron emission tomography/ computerized tomography [9].

Once the pulmonary artery aneurysm has been diagnosed, therapeutic management is first based on interventional radiology for selective vascular occlusion of the pulmonary artery responsible for hemoptysis during pulmonary angiography [1].

A pulmonary angiography is therefore used to confirm the diagnosis and perform therapeutic embolization [2]. Endovascular treatment and surgical lobectomy are the treatment options for Rasmussen's aneurysm. Because surgical lobectomy for patients with massive hemoptysis poses a high risk, endovascular treatment has become a widespread initial therapy. However, Rasmussen's aneurysm requires special diagnosis and therapeutic care, owing to its characteristic hemodynamics. The inflammation from infectious lung disease-related PAP can induce bronchial to pulmonary arterial shunt; the flow direction in this shunt is determined by the pressure gradient from the bronchial to the pulmonary artery [6]. The resulting hypo perfusion in the diseased pulmonary segment can affect visualization of the aneurysm on pulmonary angiography [10].

Several embolic materials, such as gelatin sponge, coils, and NBCA, had been shown to be effective treatment options [11,12]. Among these, n-butyl-2-cyanoacrylate (NBCA) may be the most feasible material to use, because its liquid form in an appropriate concentration enables embolization of both inflow and outflow arteries, including the aneurysm, and the risk of rupture during filling of the aneurysmal sac may be less, compared with that using coils. However, NBCA is a highly operator-dependent material to use and its behavior is difficult to estimate, especially its retrograde flow from the bronchial to the pulmonary artery. Consequently, nontarget embolization can pose dangerous and serious lung ischemic complications [13].

Aneurysm embolization equipment varies from team to team, some centers report the use of biological glue, detachable balloons, covered stents or even sclerosing agents such as the accolade [14].

Post-embolization complications remain low, with a risk of aneurysm rupture during endovascular surgery, sometimes fatal, pulmonary infarction and rare cases of gas embolism have been reported. After embolization, there is a risk of hemorrhagic recurrence in the case of aneurysmal renormalization as in our case. Surgical removal will only be considered as a last resort [2].

Remy et al., have shown that this technique is safe and effective [6,15] while surgical management in the emergency room is associated with $50 \%$ of cases of postoperative complications and $20 \%$ mortality $[16,17]$.

Khalil A et al., in their study of 189 patients who underwent treatment by endovascular means noted 13 patients to have hemoptysis of pulmonary arterial origin. Four of these patients of active tuberculosis were treated by systemic artery embolization as a first treatment, but hemoptysis recurred in all of them within a week, which was managed by specific pulmonary artery vasoocclusion [8].

\section{Conclusion}

Nowadays, Rasmussen's aneurysm has become rare thanks to the progress of antibiotic therapy; nevertheless, this diagnosis must evoke before hemoptysis of great abundance and especially in countries with a high prevalence of tuberculosis. At the same time, specific treatment must be undertaken immediately after the diagnosis of tuberculosis, as in our case.

\section{References}

1. Basille D, Andréjak C, Gosset M, Renard C, Jounieaux V (2010) Massive hemoptysis on Rasmüssen aneurysm [Hémoptysie massive sur anévrisme de Rasmüssen]. Rev Mal Respir 27: 63-6.

2. Portron Y, Lederlin M, Montaudon M, Corneloup O, Latrabe V, et al. (2010) Embolization of a Rasmussen's aneurysm complicating active tuberculosis [Embolisation d'un faux-anévrysme de Rasmussen compliquant une tuberculose active] J Radiol 91: 911-4.

3. Auerbach O (1939) Pathology and Pathogenesis of Pulmonary Arterial Aneurysm in Tuberculous Cavities. Am Rev Tuberc 39: 99-115.

4. Sanyika C, Corr P, Royston D, Blyth DF (1999) Pulmonary angiography and embolization for severe hemoptysis due to cavitary pulmonary tuberculosis. Cardiovasc Intervent Radiol 22: 457-60.

5. Plessinger VA, Jolly PN (1949) Rasmussen's Aneurysms and Fatal Hemorrhage in Pulmonary Tuberculosis. Am Rev Tuberc 60: $589-603$.

6. Remy J, Lemaitre L, Lafitte JJ, Vilain MO, Saint Michel J, et al. (1984) Massive hemoptysis of pulmonary arterial origin: diagnosis and treatment. Am J Roentgenol 143: 963-9.

7. Sbano H, Mitchell AW, Ind PW, Jackson JE (2005) Peripheral pulmonary artery pseudoaneurysms and massive hemoptysis. Am J Roentgenol 184: 1253-9.

8. Khalil A, Parrot A, Nedelcu C, Fartoukh M, Marsault C, et al. (2008) Severe Hemoptysis of Pulmonary Arterial Origin: Signs and Role of Multidetector Row CT Angiography. Chest 133: 212-9.

9. Rao VVSP, Naidu S, Reddy GV, Hanumanthu S (2018) Serendipitous Unearthing of Silent Multiple Giant Rasmussen's Aneurysms by Fluorine-18 Fluorodeoxyglucose Positron Emission Tomography/Computerized Tomography. Indian J Nucl Med 33: 136-9.

10. Sanyika C, Corr P, Royston D, Blyth DF (1999) Pulmonary angiography and embolization for severe hemoptysis due to cavitary pulmonary tuberculosis. J Vasc Interv Radiol 22: 457-60.

11. Shin S, Shin TB, Choi H, Cho JS, Kim YH, et al. (2010) Peripheral Pulmonary Arterial Pseudo aneurysms: Therapeutic Implications of Endovascular Treatment and Angiographic Classifications. Radiol 256: 656-64.

12. Tsukada J, Hasegawa I, Torikai H, Sayama K, Jinzaki M, et al. (2015) Interventional Therapeutic Strategy for Hemoptysis Originating from Infectious Pulmonary Artery Pseudoaneurysms. J Vasc Interv Radiol 26: 1046-51. 
13. Ugajin A, Fujii H, Nakamura H, Fujita A, Sasaki T, et al. (2019) Transcatheter Proximal Coil Blocking with n-Butyl-2-Cyanoacrylate Injection via the Pulmonary Artery Alone for Rasmussen's Aneurysm. J Radiol Case Rep 10.1155/2019/1725238.

14. Ramakantan R, Bandekar VG, Gandhi MS, Aulakh BG, Deshmukh HL (1996) Massive hemoptysis due to pulmonary tuberculosis: control with bronchial artery embolization. Radiolo 200: 691-4.

15. Remy-Jardin M, Wattinne L, Remy J (1991) Transcatheter occlusion of pulmonary arterial circulation and collateral supply: failures, incidents, and complications. Radiol 180: 699-705.

16. Conlan AA, Hurwitz SS, Krige L, Nicolaou N, Pool R (1983) Massive hemoptysis. Review of 123 cases. J Thorac Cardiovasc Surg 85: 120-4.

17. McCollum WB, Mattox KL, Guinn GA, Beall Jr AC (1975) Immediate Operative Treatment for Massive Hemoptysis. Chest 67: 152-5. 